\title{
Drift of Domain Walls in a Harmonic Magnetic Field
}

\author{
L. A. Pamyatnykh, A. V. Druzhinin, M. S. Lysov, S. E. Pamyatnykh, and G. A. Shmatov \\ B.N. Yeltsin Ural Federal University, Yekaterinburg, 620002 Russia \\ e-mail: Lidia.Pamyatnykh@usu.ru
}

\begin{abstract}
It is shown that a two-step form of the dynamic magnetization curve (and the hysteresis loop) established for a multiaxial ferrite-garnet wafer with a low quality factor $(Q<1)$ and considerable anisotropy in the plane $\left(K_{p} / K_{u}=14\right)$ in the frequency range of $25-1000 \mathrm{~Hz}$ is explained by the reconstruction of the dynamic domain structure, particularly by the established features of the drift of domain boundaries in the harmonic magnetic field.
\end{abstract}

DOI: $10.3103 / \mathrm{S} 1062873813100250$

\section{INTRODUCTION}

The results from simultaneously investigating the reconstruction of a domain structure (DS) and magnetooptical hysteresis loops in the region of frequencies and amplitudes of a variable magnetic field in which the drift of domain walls was observed were published in [1-3]. A harmonic magnetic field with a frequency of $25-1000 \mathrm{~Hz}$ and amplitude $H_{0}$ of up to 750 Oe was applied perpendicular to wafer plane (111) of a single ( $\mathrm{TbErGd})_{3} \mathrm{Fe}_{5} \mathrm{O}_{12}$ ferrite-garnet crystal $50 \mu \mathrm{m}$ thick with quality factor $Q<1$. The magnetic anisotropy of the sample was characterized by its cubic, induced uniaxial, and orthorhombic components. The characteristics of the sample under study are presented in the table. The domain structure was revealed using the Faraday magnetooptical effect and detected with a high-speed camera. The recording rate was 2000 frames per second.

\section{STATIC AND DYNAMIC REMAGNETIZATION OF THE FERRITE-GARNET (111) WAFER}

Figure 1a show our static (curve 1) and dynamic (curve 2) magnetooptical hysteresis loops. The dynamic hysteresis loop was measured at $f=250 \mathrm{~Hz}$ and $H_{0}=720$ Oe. One feature of the static and dynamic hysteresis loops is their two-step form, which is explained by the character of DS reconstruction in various ranges of the magnetic fields. The change in the sample DS in a constant magnetic field oriented perpendicular to the wafer's plane along axis $[\overline{1} \overline{1} \overline{1}]$ is presented in Figs. 2a-2e. Figure $2 \mathrm{f}$ gives the initial distribution of magnetization vectors $\vec{I} s$ in the sample. In the initial state (Fig. 2a), strip DS is observed in the sample after degaussing in an alternate field with a frequency of $50 \mathrm{~Hz}$ and a gradually diminishing amplitude. In this DS, the average domain width is $0.1 \mathrm{~mm}$, the domain walls (DWs) are lined up along axis [2 11$]$, while the magnetization vectors in domains $\vec{I} s$ are oriented along axes [ $\overline{1} 11]$ (light domains) and [1 $\overline{1} \overline{1}]$ (dark domains). Yellow and dark-red strips, respectively, were observed in the colored DS pattern. The DWs shifted in magnetic field $H$ parallel to axis [ $[\overline{1} \overline{1}]$ (Fig. 2b), and a single-domain state was observed at $H \geq 80$ Oe in the center of the sample (Fig. 2c); this single-domain state, in which $\vec{I} s$ was parallel to axis [1 $\overline{1} \overline{1}]$, was retained up to $H=155 \mathrm{Oe}$. At $H>155 \mathrm{Oe}$, a new magnetic phase in the form of narrow strip domains lined on average along axis [ $\overline{2} 11]$ (Fig. $2 d$ ), in which vector $\vec{I} s$ was oriented along the field (i.e., along axis $[\overline{1} \overline{1} \overline{1}]$ was formed. As the magnetic field strength grew, the volume of this phase increased (Fig. 2d); at $H>370$ Oe, a single-domain state associated with our approaching magnetic saturation was observed in the sample. In the colored DS pattern, the magnetic phase with orientation $\vec{I} s$ along the field (along axis $[\overline{1} \overline{1} \overline{1}]$ ) was green. During the remagnetization of the sample, the above stages were observed, but in reverse.

Characteristics of a (111) wafer of $(\mathrm{TbErGd})_{3} \mathrm{Fe}_{5} \mathrm{O}_{12}$ ferrite-garnet.

\begin{tabular}{c|c|c|c|c|c|c|c}
\hline$L, \mu \mathrm{m}$ & $J_{s}, \mathrm{Gs}$ & $K_{1}, \mathrm{erg} / \mathrm{cm}^{3}$ & $K_{u}, \mathrm{erg} / \mathrm{cm}^{3}$ & $K_{p}, \mathrm{erg} / \mathrm{cm}^{3}$ & $K_{1} / K_{u}$ & $K_{p} / K_{u}$ & $Q$ \\
\hline 50 & 19 & $-1.25 \times 10^{3}$ & $0.9 \times 10^{3}$ & $12.7 \times 10^{3}$ & 1.4 & 14 & 0.4 \\
\hline
\end{tabular}

$L$ is the wafer thickness, $J_{S}$ is the saturation magnetization, $K_{1}$ is a constant of cubic magnetic anisotropy, $K_{u}$ is a constant of induced uniaxial anisotropy, $K_{p}$ is a constant of orthorhombic anisotropy, and $Q$ is a quality factor. 


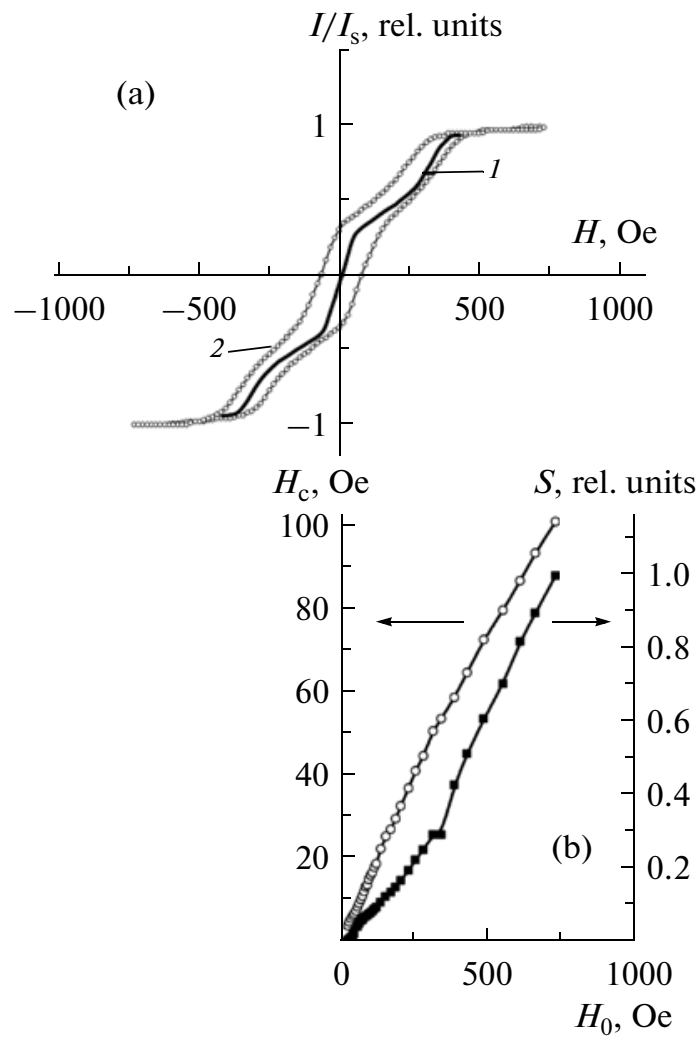

Fig. 1. (a) Statistical (curve 1) and dynamic (curve 2) magnetooptical hysteresis loops and (b) the dependence of the area of dynamic hysteresis loops $S$ and coercive force $H_{0}$ on the amplitude of variable magnetic field $H_{\mathrm{c}}$. Field frequency $f=250 \mathrm{~Hz}$.

We may conclude from a comparison of our visual observations of the DS and the form of the static hysteresis loop that the first inflection point in the loop corresponds to $H=83 \mathrm{Oe}$, at which the intermediate single-domain state is observed (Fig. 2c). The second inflection point in the loop corresponds to the region of the fields in which the sample approaches the single-domain state with magnetization vector $\vec{I} s$ parallel to the field.

Figure $1 \mathrm{~b}$ represents the dependences of the area of dynamic hysteresis loops $S$ and coercive force $H_{\mathrm{c}}$ on the amplitude of the alternate field at frequency $f=$ $250 \mathrm{~Hz}$. The abrupt increase in the coercive force and the area of dynamic hysteresis loops indicates an rise in losses for the remagnetization of the sample upon increasing the amplitude of the alternate magnetic field. An increase in total losses for the remagnetization of $\mathrm{Fe}-3 \% \mathrm{Si}$ crystals with an increase in the

Fig. 2. Domain structure of a $\overrightarrow{(111) \text { wafer } \text { of }}$ $(\mathrm{TbErGd})_{3} \mathrm{Fe}_{5} \mathrm{O}_{12}$ ferrite-garnet in a constant magnetic field oriented perpendicular to the sample plane and equal to (a) 0 , (b) 30 , (c) 153 , (d) 297 , (e) $354 \mathrm{Oe}$; (f) model of the strip domain DS in the initial degaussed state.
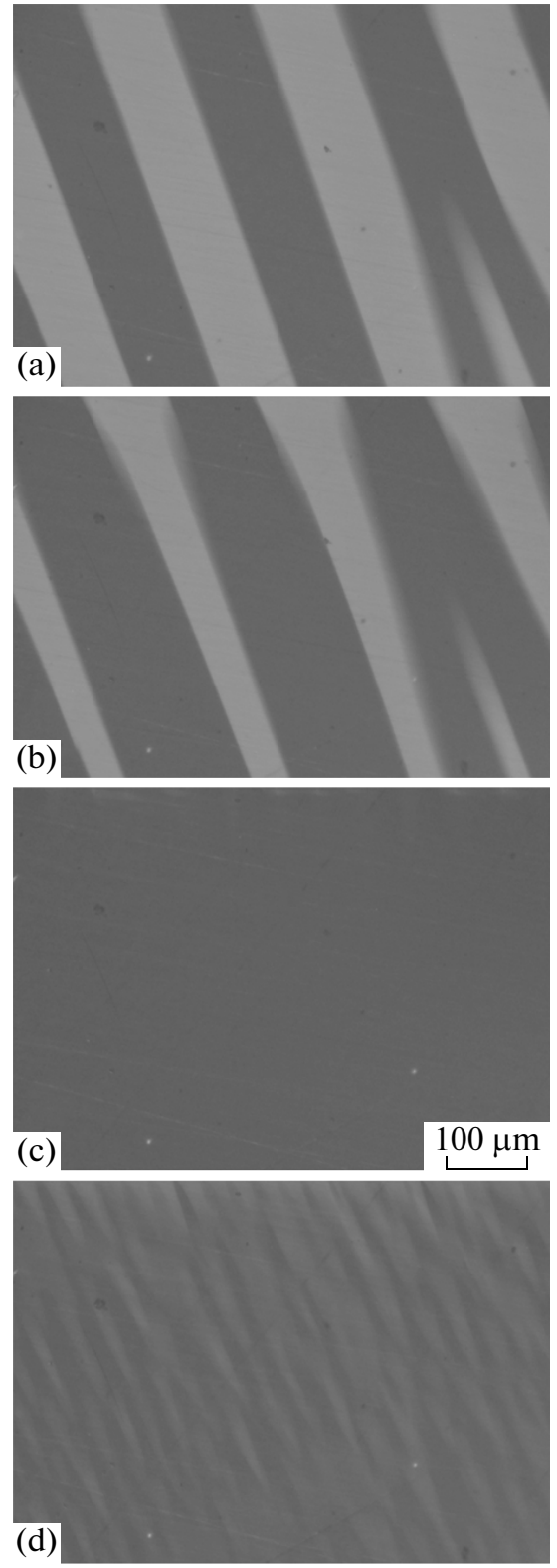

(e)

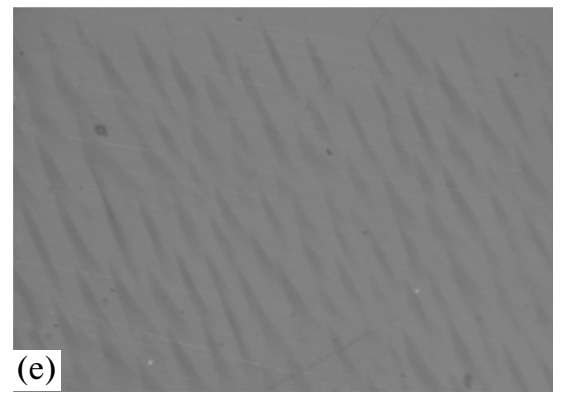

(f)

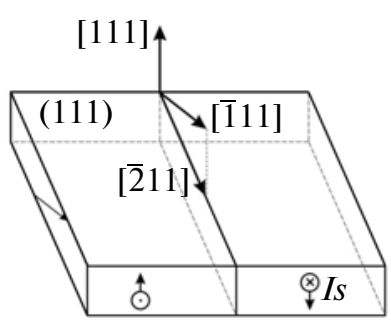


induction of the alternate magnetic field in the region of its low amplitudes was reported in [4].

\section{DRIFT OF DOMAIN BOUNDARIES}

We determined the region of DW drift in the frequency-amplitude coordinates of the alternate sinusoidal magnetic field and found the dependences of the drift velocity of domain walls $V$ on amplitude $H_{0}$ at fixed frequency $f$.

In the frequency range of $25-1000 \mathrm{~Hz}$, the drift starts in the field with amplitude $H_{0} \geq 30$ Oe. Up to $H_{0}=60 \mathrm{Oe}$, the pure drift for which the strip domain structure is retained over all the field period is observed. Observations show that as the field amplitude increases, the domain width increases with magnetization over the field, and at $H_{0}=150$ Oe the sample is in a single-domain state for a larger part of the field period. The drift velocity in the range of field amplitudes $60<H_{0}<130$ Oe was thus measured for that part of the field period in which the integrity of the strip DS was retained.

Figure 3 presents the dependences of the period of the strip domain structure $d$ (curve 1 ) and the drift velocity of domain boundaries $V$ (curve 2) on the amplitude of the variable magnetic field (Fig. 3a) and the initial portion of the dynamic magnetization curve constructed from the limiting values of the coordinates at the tops of dynamic hysteresis loops (Fig. 3b) for field frequency $f=250 \mathrm{~Hz}$. Figure $3 \mathrm{c}$ gives the $H_{0}$ dependences of coercive force $H_{\mathrm{c}}$ and the area of dynamic hysteresis loops $S$ for the field amplitudes at which the DW drift was observed in the sample. It can be seen that the character of the dependence of the drift DW velocity on the field amplitude changes at $H_{0}=60$ Oe (Fig. 3a). The inflection point in the dynamic magnetization curve is observed precisely at this amplitude of the alternate field (Fig. 3b). The same field region corresponds to variations in the character of the dependence of the area of dynamic hysteresis loops, the inflection point in dependence $H_{\mathrm{c}}\left(H_{0}\right)$ (Fig. 3c), and a decline in structure period $d$ (curve 1 in Fig. 3a).

The correlation between varying the drift DW velocity and the form of the dynamic magnetization curve could indicate the influence of DW drift on the system's integral magnetic characteristics.

Direct observations of the reconstruction of the domain structure in a variable magnetic field along the hysteresis loop showed that the hysteresis was caused by two factors: (i) the delayed emergence and growth of the new magnetic phase in the variable magnetic field and (ii) the abrupt increase in the number of magnetic dislocations [2] upon increasing the amplitude of the harmonic magnetic field.
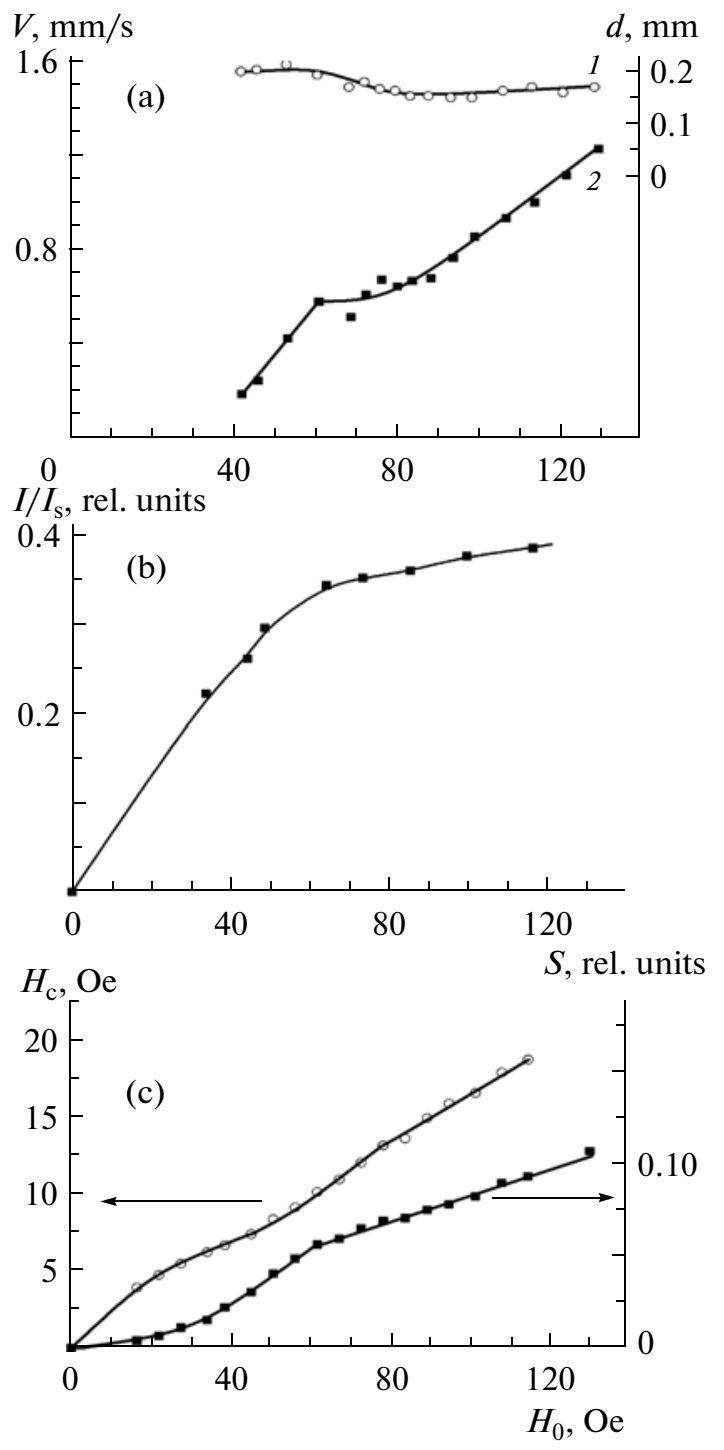

Fig. 3. Dependences on amplitude $H_{0}$ of a variable magnetic field with frequency $f=250 \mathrm{~Hz}$ (a) for the period of the strip domain structure $d$ (curve 1 ) and drift velocity $V$ (curve 2), (b) for the relative magnetization (the initial segment of the dynamic magnetization curve $I\left(H_{0}\right) / I_{\mathrm{s}}$, and (c) for the area of dynamic hysteresis loops $S$ and coercive force $H_{\mathrm{c}}$ (the initial segments of the corresponding curves in Fig. 1b).

\section{NUMERICALLY MODELING THE MOTION OF DOMAIN BOUNDARIES}

It was shown in [5] that under certain conditions, the translational motion of the strip domain structure in an external oscillating magnetic field is possible. This result in [5] was found for large oscillating frequencies of a magnetic field comparable to the frequency of proper oscillations of the DW system $\left(\sim 10^{8} \mathrm{~Hz}\right.$ for the materials under study), and the motion of the DW system under the effect of the oscillating magnetic field was due to the asymmetry of the initial velocities of neighboring DWs. 


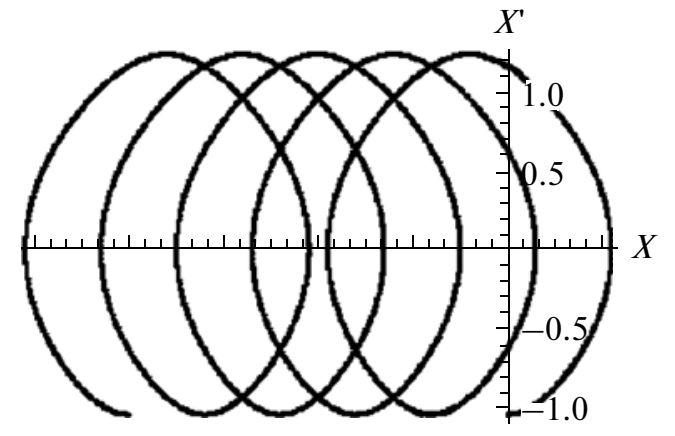

Fig. 4. Phase portrait of oscillations of one domain boundary upon the motion of strip DS as a whole. Along the axes, $X$ is the coordinate of the domain boundary and $X^{\prime}$ is the velocity of strip DS as a whole (all in relative units). Amplitude of the external magnetic field $h=0.5$ of the saturation field, and its circular frequency $\omega=2 \pi f$ and $f=250 \mathrm{~Hz}$.

Based on the approach developed in [5], we numerically modeled the motion of the system of domain walls of the strip domain structure in a uniaxial wafer of $(\mathrm{TbErGd})_{3}(\mathrm{FeAl})_{5} \mathrm{O}_{12}$ ferrite-garnet placed into an oscillating external magnetic field oriented perpendicular to its plane. The parameters of the sample were $I_{\mathrm{s}}=40 \mathrm{Gs}$ and $K_{u} / K_{1}=1.6$. Modeling showed that movement of the strip domain structure as a whole was possible at frequencies of $\sim 10^{2} \mathrm{~Hz}$, i.e., in the region where the drift of the DW system was observed in this sample experimentally.

Figure 4 shows the phase portrait of oscillations of one domain boundary of the strip domain. It can be seen that the boundary responsible for the oscillations additionally moves translationally, and the coordinate of the domain wall shifts through the field period (with the conservation of DW velocity) to the same magnitude.

\section{CONCLUSIONS}

A correlation between the dynamic behavior of the domain structure and the form of the dynamic magnetization curve (and the hysteresis loop) was established for a multilayered ferrite-garnet wafer with a low quality factor $(Q<1)$ and considerable anisotropy in plane $\left(K_{p} / K_{u}=14\right)$. It was shown that the two-step form of the dynamic magnetization curve (and the hysteresis loop) in a frequency range of $25-1000 \mathrm{~Hz}$ is explained by the behavior of the dynamic domain structure, particularly by the established features of the drift of domain walls in a harmonic magnetic field.

The drift of domain boundaries was numerically modeled for a uniaxial ferrite-garnet wafer. Our modeling showed that drift was possible at frequencies on the order of $10^{2} \mathrm{~Hz}$, i.e., in the region where DW drift was observed experimentally.

\section{REFERENCES}

1. Vlasko-Vlasov, V.K. and Uspenskaya, L.S., Zh. Eksp. Teor. Fiz., 1986, vol. 91, no. 4, p. 1483.

2. Pamyatnykh, L.A., Lysov, M.S., and Kandaurova, G.S., Bull. Russ. Acad. Sci. Phys., 2007, vol. 71, no. 11, p. 1497.

3. Pamyatnykh, L.A., Lysov, M.S., Shmatov, G.A., Kandaurova, G.S., and Druzhinin, A.V., Bull. Russ. Acad. Sci. Phys., 2010, vol. 74, no. 10, p. 1417.

4. Tiunov, V.F. and Filippov, B.N., Fiz. Met. Metalloved., 2006, vol. 102, no. 3, p. 280.

5. Solov'ev, M.M. and Filippov, B.N., Fiz. Met. Metalloved., 2004, vol. 98, no. 3, p. 12.

Translated by N. Korovin 